\title{
The use of trans-applicator intracavitary ultrasonography in brachytherapy for cervical cancer: phantom study of a novel approach to 3D image-guided brachytherapy
}

\author{
Tomoaki Tamaki, MD, PhD1,2,3, Kazunori Miyaura, MS², Toshihiro Murakami, BS², Yu Kumazaki, PhD², \\ Yoshiyuki Suzuki, MD, PhD', Takashi Nakano, MD, PhD³, Shingo Kato, MD, PhD² \\ 'Department of Radiation Oncology, Fukushima Medical University, Fukushima, Fukushima, ${ }^{2}$ Department of Radiation Oncology, Saitama \\ Medical University International Medical Center, Hidaka, Saitama, ${ }^{3}$ Department of Radiation Oncology, Gunma University Graduate School \\ of Medicine, Maebashi, Gunma, Japan
}

\begin{abstract}
Purpose: To assess the feasibility of applying trans-applicator intracavitary ultrasonography to image-guided brachytherapy for cervical cancer.

Material and methods: For this experiment, a phantom was created and included a polyethylene tube, intended to simulate a tandem applicator, which was inserted into chicken meat and embedded in agar, along with magnetic resonance imaging (MRI)-compatible ovoid applicators. Three-dimensional images of the phantom were obtained using computed tomography (CT), MRI (T2-weighted), and intracavitary ultrasonography sectional images acquired at $1 \mathrm{~mm}$ slice intervals. Intracavitary ultrasonography images were acquired from within the simulated tandem applicator using a radial transducer. Magnetic resonance imaging and intracavitary ultrasonography images were manually registered onto CT images. The chicken meat was contoured as the target volume independently on the CT, MRI, and intracavitary ultrasonography images, and the Dice similarity coefficient was used to compare the target volumes. The dose distributions of a sample brachytherapy plan were also evaluated.

Results: Computed tomography, MRI, and intracavitary ultrasonography all visualized the three-dimensional phantom volumes. Intracavitary ultrasonography images depicted the meat with high echoic signals and a border clearly distinguishable from the surrounding agar. The Dice similarity coefficient values for the target volumes on CT vs. MRI, CT vs. intracavitary ultrasonography, and MRI vs. intracavitary ultrasonography were $0.966,0.965$, and 0.971 , respectively, indicating similar contouring with the three modalities. Among the modalities, the differences in $\mathrm{D}_{50}, \mathrm{D}_{90}$, $\mathrm{D}_{98}$, and $\mathrm{D}_{100}$ values were $1.8 \%, 2.9 \%, 3.7 \%$, and $2.9 \%$, respectively.

Conclusions: Three-dimensional reconstructed trans-applicator intracavitary ultrasonographic images clearly depicted meat tissue within the phantom, and could thus be used for brachytherapy planning. This study proves the concept of trans-applicator intracavitary ultrasonography for intracavitary brachytherapy. Further research such as development of intracavitary ultrasonography system, 3D reconstruction method, ultrasonography-compatible applicators, and ultrasonography-based target concept is warranted to assess the potential clinical application.
\end{abstract}

J Contemp Brachytherapy 2017; 9, 2: 151-157 DOI: https://doi.org/10.5114/jcb.2017.66588

Key words: brachytherapy, cervical cancer, intracavitary ultrasonography.

\section{Purpose}

Brachytherapy plays a vital role in radiation therapy for cancer, and currently cervical cancer treatment is one of the largest applications for this modality. Technological advancements have led to the inclusion of threedimensional (3D) imaging modalities such as computed tomography (CT) and magnetic resonance imaging (MRI) in brachytherapy treatment planning [1,2]. Thus, 3D image-guided brachytherapy (IGBT) has yielded improvements in disease control $[3,4]$ and the avoidance of adverse effects $[5,6,7]$. On a global scale, high incidence rates of cervical cancer have been reported in countries with low and medium national income levels [8]. Although some studies have indicated that 3D IGBT is cost-effective when compared to conventional brachytherapy practices that

\footnotetext{
Address for correspondence: Tomoaki Tamaki, MD, PhD, Department of Radiation Oncology, Fukushima Received: 07.10 .2016 Medical University, 1 Hikarigaoka, Fukushima, Fukushima 960-1295, Japan, phone: +81 245471630 , 
use two-dimensional fluoroscopic images [9], the significant patient burden associated with cervical cancer and relatively high economic cost of introducing $\mathrm{CT}$ and/or MRI present challenges to the implementation of 3D IGBT.

Ultrasonography is a relatively widespread and affordable modality used to acquire tomographic images, and it has been widely applied for the diagnosis of abdominal and gynecologic diseases. The use of trans-rectal ultrasonography (TRUS) in the context of brachytherapy for prostate cancer is a well-known practice, as is the use of ultrasonography for dosimetric analyses of implanted radioactive iodine seeds [10], and for image guidance during needle applicator insertions for high dose rate interstitial brachytherapy [11]. The use of trans-abdominal ultrasonography (TAUS) [12,13] and TRUS [14] has also been reported in the context of brachytherapy for cervical cancer.
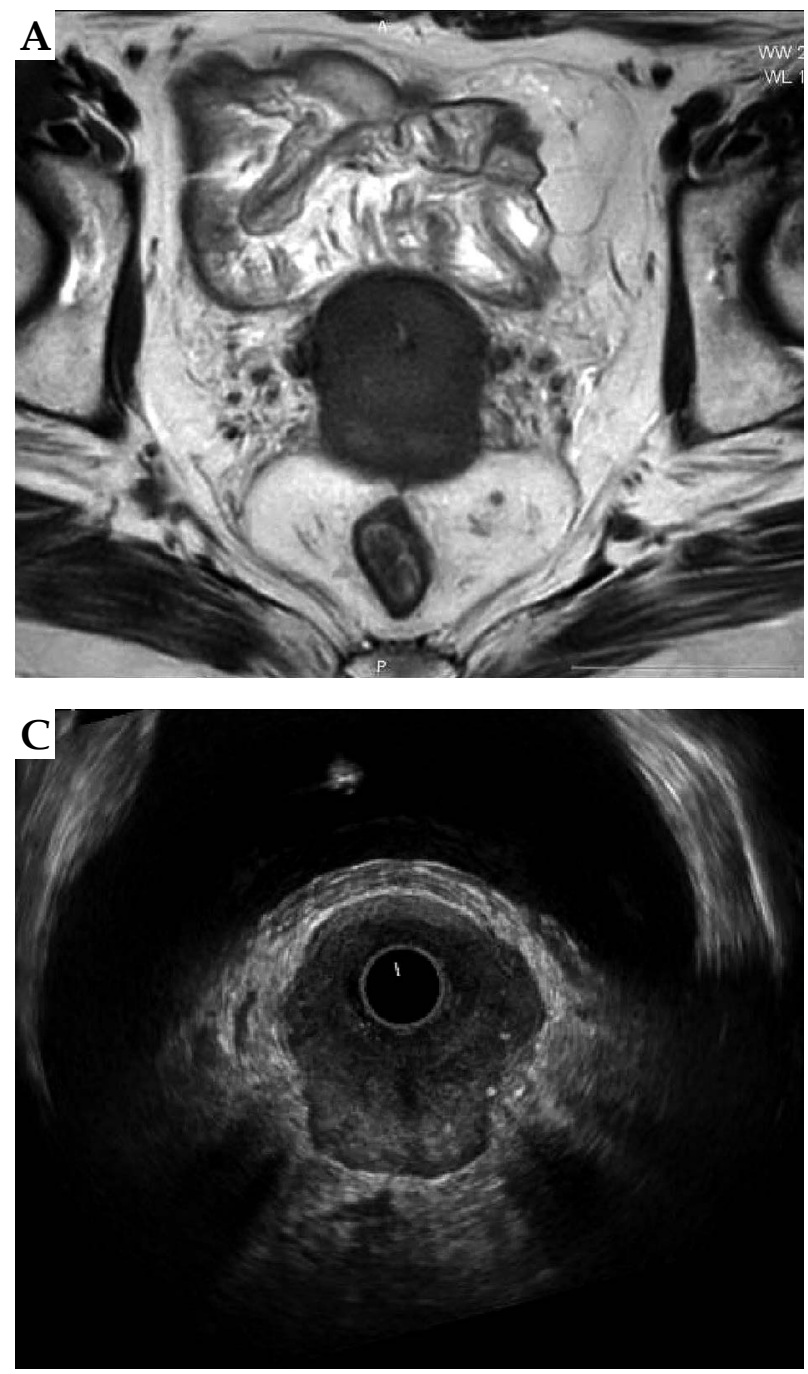

In our clinical experience with ultrasonography for cervical cancer, one of the most effective methods is image acquisition from the uterine cavity or uterine canal using an intracavitary ultrasonography (ICUS) transducer. In the case shown in Figure 1, ICUS was used to examine a shrinking cervical tumor during the weekly intracavitary/interstitial brachytherapy sessions. Other reports also showed that cervical cancer lesions continuously regress during radiotherapy $[15,16]$. Intracavitary ultrasonography provided real-time images with clear soft-tissue contrast at the time of brachytherapy, and were occasionally more useful than the diagnostic MRI image acquired prior to the first brachytherapy session. These experiences have led us to explore the use of ICUS in intracavitary brachytherapy for cervical cancer.

In this study, we used a phantom to test the feasibility of obtaining 3D data from ICUS images acquired
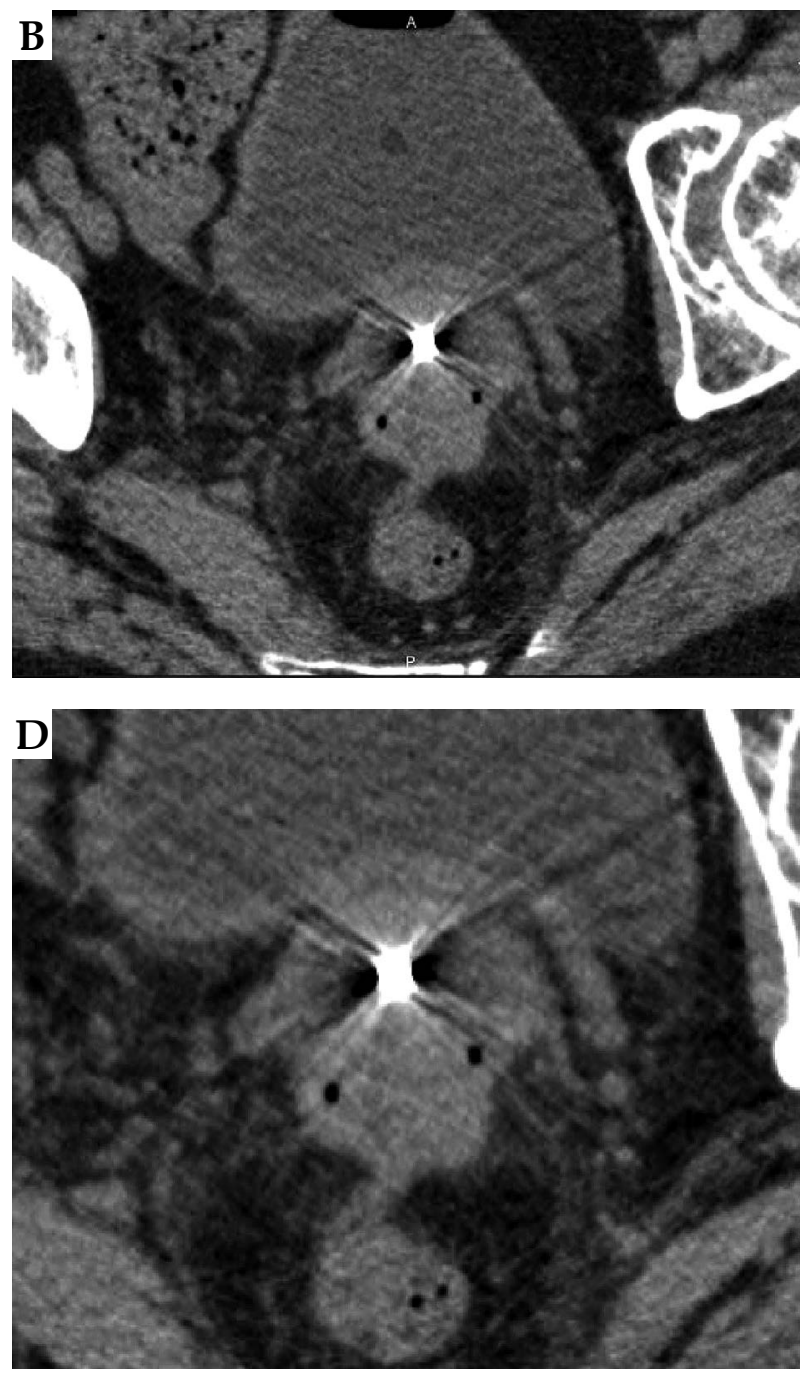

Fig. 1. A case involving weekly intracavitary/interstitial brachytherapy sessions, in which intracavitary ultrasonography was useful. In our practice, we routinely perform pre-brachytherapy diagnostic magnetic resonance imaging (MRI) (A) for reference during brachytherapy sessions. Because the shape and size of the primary tumor changes during the treatment course, computed tomography (CT) images obtained concurrently with later brachytherapy sessions (B) might not fully correlate with the pre-brachytherapy MRI data (A). The use of intracavitary ultrasonography during the brachytherapy session (C) provided a better depiction of the structures around the cervical tumor and became a good reference for CT-based brachytherapy (D, enlarged image of B) 
from within an intrauterine tandem applicator and using these data for intracavitary treatment planning. The phantom was imaged using CT, MRI, and transapplicator ICUS. Target delineation and dose distributions were compared among the 3D images obtained with the three modalities. This study demonstrates feasibility of using 3D information acquired via ICUS to brachytherapy for cervical cancer.

\section{Material and methods \\ Brachytherapy phantom}

A phantom was created for this experiment. A piece of chicken meat, into which a polyethylene tube had been inserted, was embedded in agar in a plastic container. Although the outer and inner diameters of the tube were $15 \mathrm{~mm}$ and $13 \mathrm{~mm}$, respectively, and were relatively larger than diameters of actual tandem applicators used clinically, the tube was used to simulate the intrauterine tandem applicator for this study. MRI-compatible ovoid applicators were also embedded into the agar to simulate intravaginal applicators.

\section{Computed tomography and magnetic resonance image acquisition}

Axial CT images of the phantom were obtained at $1 \mathrm{~mm}$ slice thickness using the same large-bore CT scanner (Aquilion LB; Toshiba, Tokyo, Japan) used for image-guided brachytherapy treatment at our institute, as well as the sequence used clinically for the pelvic imaging $(135 \mathrm{kV}, 250 \mathrm{~mA}$, displayed field of view [DFOV] of $550 \mathrm{~mm}$, gantry rotation of $0.5 \mathrm{~s}$ ). T2-weighted MRI images were acquired using the $3 \mathrm{D}$ fast spin-echo technique (FSE) on a 3-T system (Philips Achieva dStream; Koninklijke Philips N.V., Amsterdam, The Netherlands) with a pelvic surface coil and the following settings: repetition time of 2,500 ms, echo time of $245 \mathrm{~ms}$ (DFOV of $480 \mathrm{~mm}$ ). The axial reconstruction thickness was $1 \mathrm{~mm}$, with an intersection gap of $1 \mathrm{~mm}$.

\section{Radial-transducer intracavitary ultrasonography image acquisition}

Intracavitary ultrasonography images were acquired from within the simulated tandem applicator using a diagnostic ultrasound system (Diagnostic Ultrasound System ARIETTA60; Hitachi Aloka Medical, Tokyo, Japan) with a radial transducer (R41R transducer, 0-10 MHz) (Figure 2). The radial transducer can acquire the real-time image of 360 degrees in the plane perpendicular to the axis of the transducer. The diameter of the transducer was $12.5 \mathrm{~mm}$. Intracavitary ultrasonography axial images of the phantom were acquired from the cavity of the water-filled polyethylene tube in the phantom at increments of $1 \mathrm{~mm}$. This $1 \mathrm{~mm}$ increment was measured using the detector holder from the WP1D-manual water phantom (IBA Dosimetry GmbH, Schwarzenbruck, Germany). Intracavitary ultrasonography images in DICOM format were exported from the ultrasound system and used for 3D image reconstruction.

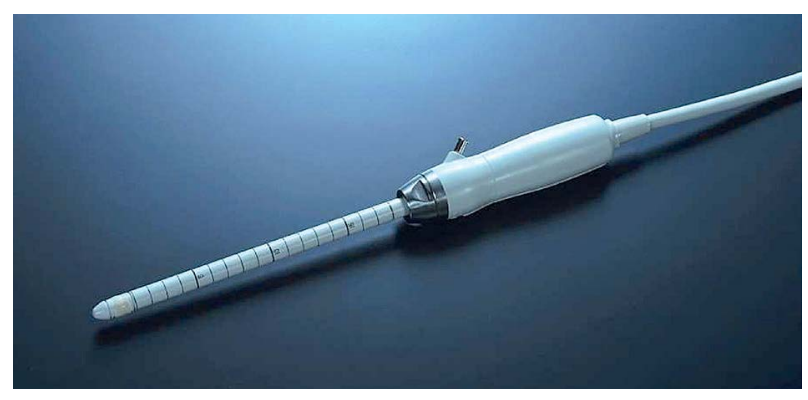

Fig. 2. Radial transducer used in this experiment

\section{D image reconstruction, image registration, and target delineation}

The 3D CT, MRI, and ICUS images were reconstructed using image processing software (MIM Maestro version 6.2, MIM Software Inc., Cleveland, OH, USA). Magnetic resonance imaging and ICUS images were visually registered onto CT images via manual rigid registration. Manual registration was performed according to the shape of the chicken meat and a gold marker $(0.9 \mathrm{~mm}$ in diameter, $3 \mathrm{~mm}$ long) that had been implanted for reference. Because ICUS images were acquired with a radial transducer, images beyond the innermost position in the polyethylene tube could not be acquired. For this experiment, the chicken meat was used as the simulated target volume and was independently delineated on the axial sections of CT, MRI, and ICUS images. Contouring was performed on the CT or MRI slices that corresponded to available slices from ICUS imaging. The Dice similarity coefficient (DSC) was used to evaluate the spatial overlap of the target volumes delineated on CT, MRI, and ICUS images.

\section{Simulated brachytherapy planning and dose-volume histogram parameters using delineated target volumes}

A sample brachytherapy plan was created on the CT images, and the dose distributions were evaluated using CT, as well as the registered MRI and ICUS images. The minimum doses delivered to $50 \%, 90 \%, 98 \%$, and $100 \%$ of the volume of interest $\left(\mathrm{D}_{50}, \mathrm{D}_{90}, \mathrm{D}_{98}\right.$, and $\mathrm{D}_{100}$, respectively) were computed and compared.

\section{Computed tomography, magnetic resonance image, and intracavitary ultrasonography image of the clinical case}

Computed tomography, MRI, and ICUS images of the clinical case described in the Purpose were acquired at Saitama Medical University International Medical Center, and the use of these images was approved by the institutional review board of Saitama Medical University International Medical Center.

\section{Results}

The 3D reconstructed CT, MRI, and ICUS images of the phantom are shown in Figure 3. On CT images, the chicken meat appeared hyperdense relative to the surrounding agar, and the border of the meat was identifiable at the 
liver window (window width of $160 \mathrm{HU}$, window level of $40 \mathrm{HU}$ ). On FSE-T2 weighted MRI, the meat exhibited a hypointense signal relative to the hyperintense signal of the agar. The border of the meat exhibited a very strong contrast with the surrounding agar, as shown in Figure 3. On ICUS images, the meat appeared as a hyperechoic area surrounded by highly hypoechoic agar. Small internal structures of the meat, such as muscle fibers, were identifiable on the ICUS images, and the border of the meat was very sharp. As shown in Figure 3, the coronal and sagittal images were reconstructed clearly from axial images with a $1 \mathrm{~mm}$ slice thickness. Given the geometric characteristics of the radial transducer for ICUS, images could not be acquired beyond the innermost position inside the simulated tandem (Figure 3). In contrast to CT and MRI, the internal structures of the ovoid applicators were not clearly visualized with ICUS.

The target volume (meat) was contoured independently on the CT, MRI, and ICUS images, and the delineated target volumes and the DSC values of comparisons among the modalities are shown in Table 1. The DSC values for CT vs. MRI, CT vs. ICUS, and MRI vs. ICUS were 0.966 , 0.965 , and 0.971 , respectively.

The dose distributions of the sample brachytherapy plan, which was created on CT images and registered onto MRI and ICUS images, are shown in Figure 4.

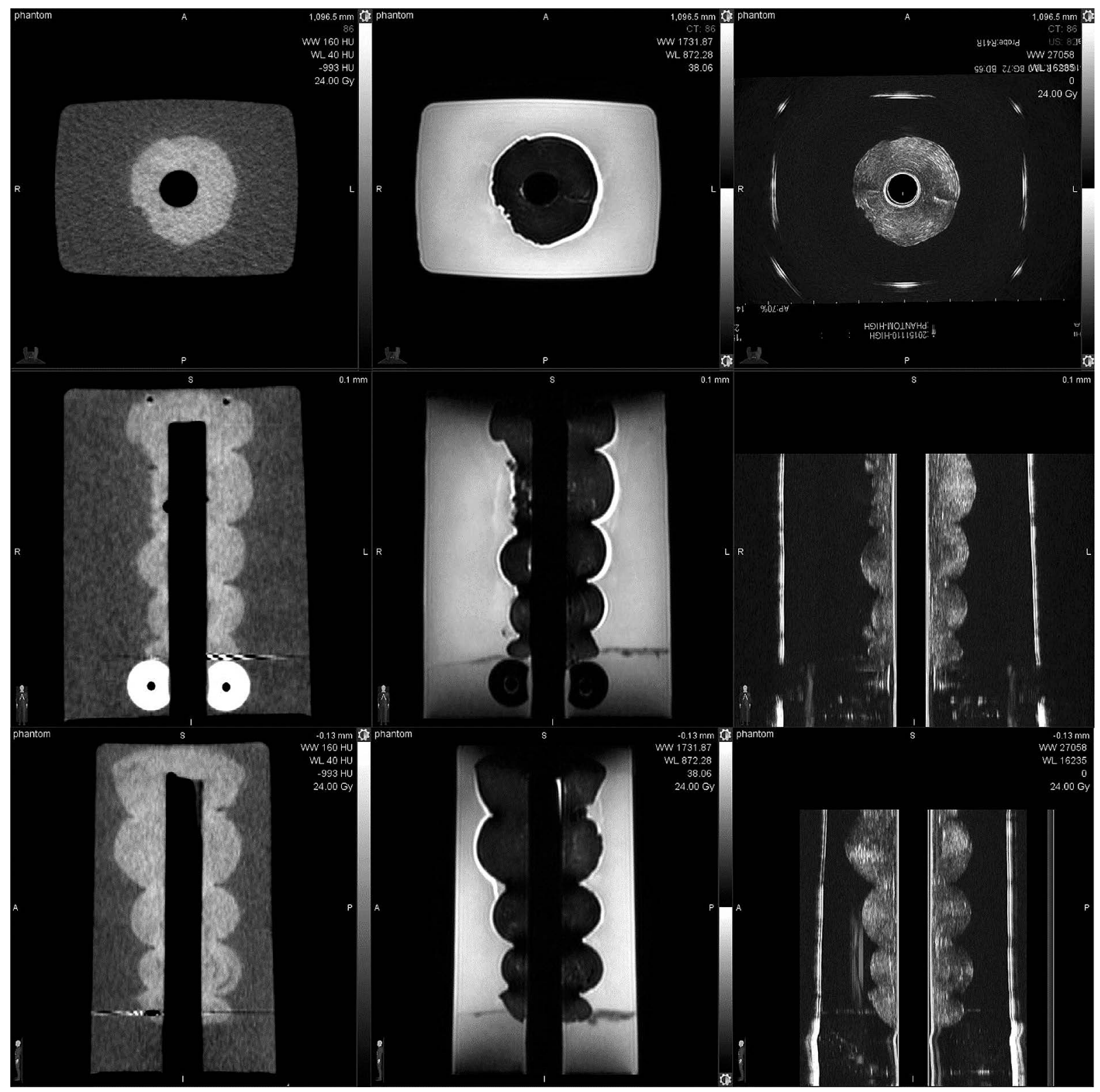

Fig. 3. Three-dimensional (3D) reconstructed computed tomography (CT) (left column), magnetic resonance imaging (MRI) (center column), and intracavitary ultrasonography (right column) images of the phantom (top row: axial images, middle row: coronal images, bottom row: sagittal images). A 3D image was successfully reconstructed using intracavitary ultrasonography images taken from the cavity of the simulated tandem applicator. The coronal and sagittal images were comparable to those obtained with CT and MRI 
Table 1. Target volumes, Dice similarity coefficients (DSC; 1 = identical), and dose-volume histogram (DVH) parameters

\begin{tabular}{|c|c|c|c|}
\hline & Computed tomography & Magnetic resonance imaging & Intracavitary ultrasonography \\
\hline Target volume (ml) & 135.51 & 140.87 & 139.82 \\
\hline \multicolumn{4}{|l|}{ DSC: CT vs. MRI } \\
\hline \multicolumn{4}{|c|}{0.966} \\
\hline \multicolumn{4}{|l|}{ DSC: CT vs. ICUS } \\
\hline & \multicolumn{3}{|c|}{0.965} \\
\hline \multirow[t]{2}{*}{ DSC: MRI VS. ICUS } & & 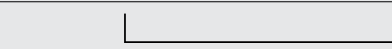 & \\
\hline & & \multicolumn{2}{|c|}{0.971} \\
\hline$D_{50}(G y)$ & 7.26 & 7.13 & 7.17 \\
\hline$D_{90}(G y)$ & 4.22 & 4.14 & 4.26 \\
\hline$D_{98}(G y)$ & 3.32 & 3.24 & 3.36 \\
\hline$D_{100}(G y)$ & 2.45 & 2.40 & 2.47 \\
\hline
\end{tabular}

$D_{50}, D_{90}, D_{98}, D_{100}$ - the minimum dose received by $50 \%, 90 \%, 98 \%, 100 \%$ of the target volume

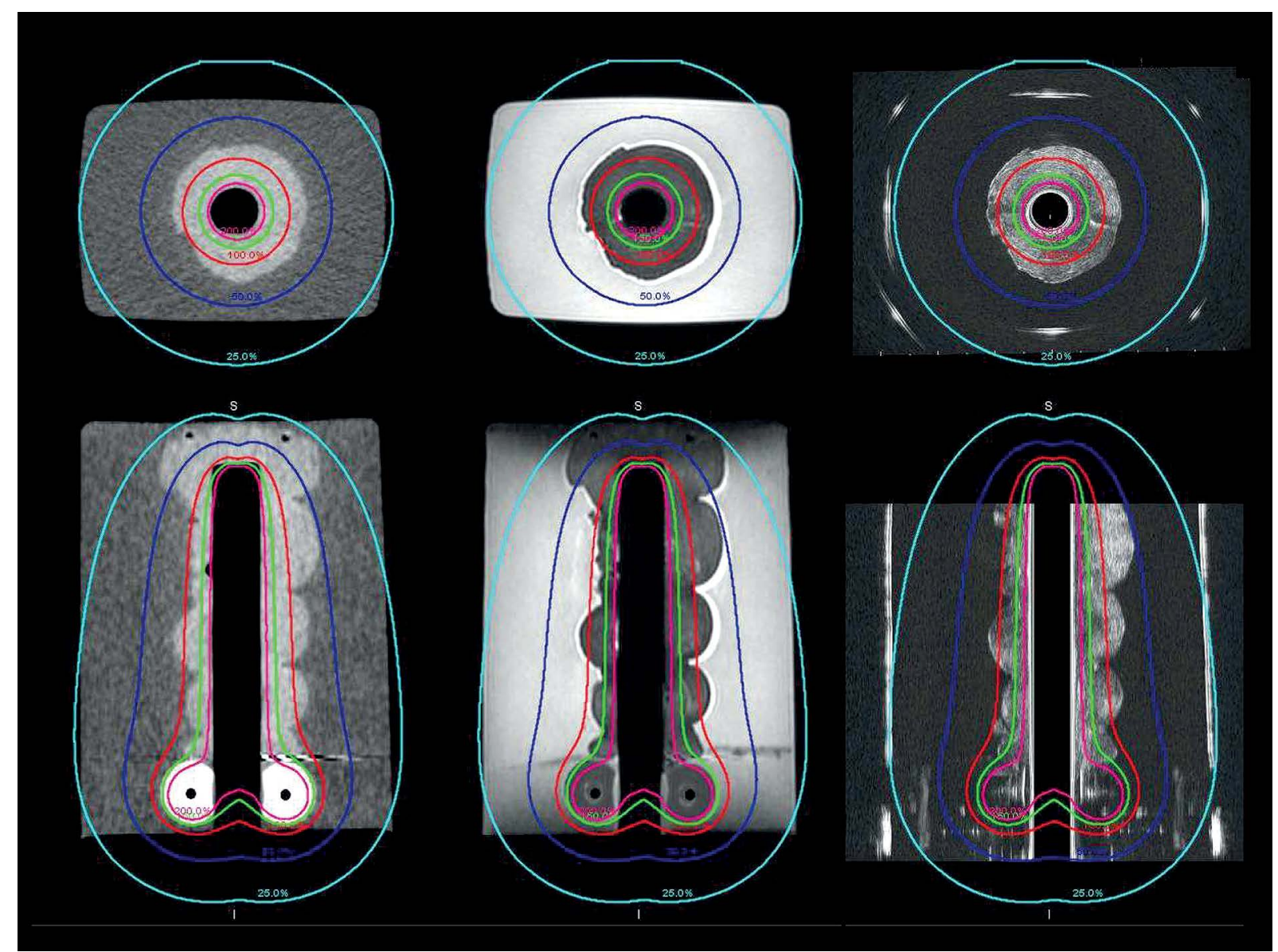

Fig. 4. Dose distributions of a sample brachytherapy plan according to computed tomography (left column), magnetic resonance imaging (center column), and intracavitary ultrasonography (right column). Dose distributions could be evaluated using visual observations and dose-volume histogram analyses (top row: axial images, bottom row: coronal images)

$\mathrm{D}_{50}, \mathrm{D}_{90}, \mathrm{D}_{98}$, and $\mathrm{D}_{100}$ values based on targets from the $\mathrm{CT}$, MRI, and ultrasound images are compared in Table 1, and the respective differences in the DVH parameters were $1.8 \%, 2.9 \%, 3.7 \%$, and $2.9 \%$, respectively.

\section{Discussion}

This study tested the feasibility of ICUS imaging from an ICBT applicator for intracavitary brachytherapy for cervical cancer. This experiment demonstrated that ICUS 
via an intracavitary brachytherapy applicator could be used to reconstruct 3D images of the applicator and its surrounding target, to identify the target for delineation, and to evaluate brachytherapy dose distributions.

Ultrasonography has been applied widely in the setting of brachytherapy for prostate cancer [10,11], but has been much more limited in the context of cervical cancer brachytherapy $[12,13,14]$. Regarding the implantation of radioactive iodine seeds in the prostate, TRUS is the primary modality used for image-guidance during implantation and treatment planning [10]. In high dose rate interstitial brachytherapy for prostate cancer, TRUS is used for image-guidance during the insertion of interstitial needle applicators and treatment planning [11]. For cervical cancer brachytherapy, applications involving both TAUS and TRUS have been reported. Trans-abdominal ultrasonography is often used to assist the insertion of an intrauterine applicator. Previous reports have demonstrated a method [12] and efficacy [13] of using sagittal and axial TAUS images to prescribe brachytherapy doses to the perimeters of cervical cancer targets (tumor, cervix, and uterine corpus). This method does not involve 3D volumetric treatment planning, but is rather an advance from two-dimensional brachytherapy planning that only utilizes orthogonal fluoroscopic images; this newer method could identify the basic target volume dimensions on orthogonal cross-sectional ultrasonography images, and adjust the target dose coverage to perform more conformal brachytherapy. The cervical and uterine corpus measurements obtained with TAUS and MRI were found to be comparable [17]. Schmidt et al. reported that TRUS could be used to identify high-risk clinical target volumes (HR-CTVs) within the intraobserver variability associated with MRI. In addition, TRUS was superior to CT in terms of HR-CTV identification [14]. Another report by Petric and Kirisits demonstrated a possible application of transcervical endosonography in brachytherapy for cervical cancer by presenting its use in the assessment of cervical tumor and surrounding organs [18]. They have shown that free-hand endocervical ultrasonographic images using a rotating ultrasonography transducer could be safely acquired at the time of brachytherapy, and that hypoechoic region on representative slices on ultrasonography corresponded well to the HR-CTV region depicted on corresponding MRI T2 images [18]. The phantom study and clinical example (Figure 1) presented in our report demonstrate the conceptual feasibility of applying 3D volumetric information from ICUS to HR-CTV identification and treatment planning evaluations. This study used 3D reconstruction of stepwise axial images acquired with a non-rotating radial (360 degrees) transducer and, along with the previous report [18], suggests a potentially new application of intracavitary/transcervical ultrasonography to the IGBT for cervical cancer.

Regarding cervical cancer imaging, ICUS can be used to acquire images that are similar in quality to those obtained with MRI, while featuring logistical and economic advantages. When considering brachytherapy applications, ICUS can have following advantages over TAUS and TRUS. The first possible advantage of ICUS is its superior image quality, which results from the proxim- ity of the transducer to the target. In TAUS, for example, the removal of gas from the bowels or appropriate filling of the bladder might be required in order to acquire a clear image of the uterus and cervix. In TRUS, imaging in the direction of uterine corpus may be difficult, given the anatomic limitation of the rectum. The second possible advantage is that because ICUS and ICBT are both intracavitary procedures implemented from the same cavity, the area visualized most clearly by ICUS corresponds to the area to which ICBT will deliver a high dose. On the other hand, the assessment of complete structure of organs at risk such as rectum and bladder may be challenging because of factors such as increasing distance from the transducer and positional changes of the patient [18]. The third possible advantage originates from the method of ICUS imaging acquisition, which is centered on the applicator. By using the applicator as a positioning reference, the image acquisition and 3D image reconstruction consistency among fractions and operators could be improved, especially in comparison to TAUS.

Regarding procedures used in actual practice, ICUS imaging from the brachytherapy applicator presents an advantage. For CT and MR image acquisition, either the patient must be transferred to the CT or MRI room with the intracavitary brachytherapy applicators already in place, or a CT or MRI scanner must be installed in the operation theater or brachytherapy room. In contrast, the ultrasonography system is sufficiently compact and mobile to be brought to the operating theater or brachytherapy room. Furthermore, ICUS from the applicator can be easily introduced into in the workflow of regular applicator insertion procedures with a minimal additional burden placed on the patient. Accordingly, ICUS may be suitable for facilities with high patient loads and those using CT because the additional workload could be minimal.

This report describes a phantom study that originated from our clinical experiences and the advantages of ICUS for cervical cancer. To fully develop this method for procedural applications in actual routine clinical practice, further efforts are needed regarding the development of applicators and a compatible ultrasonography system, including an ultrasonographic transducer small enough to fit the applicator, a 3D reconstruction method for images acquired from the possibly curved uterine cavity, and ultrasonography-compatible applicators. Further research on relationship between MRI and ICUS findings is also required to utilize ICUS in IGBT using the concept of target volumes described in ICRU Report 89. Nonetheless, this relatively easy and simple method could significantly improve the practice of brachytherapy by allowing a wide range of practitioners and patients to move toward the use of 3D image guidance.

\section{Conclusions}

A 3D reconstructed image from trans-applicator ICUS data allowed clear identification of chicken tissue within a phantom, indicating that this modality could be used to evaluate brachytherapy plans. This study provides proofof-concept for the use of trans-applicator ICUS in intracavitary brachytherapy. Although further experiments 
are warranted to assess the potential clinical applications, this methodology may represent a simple and easy method by which 3D image guidance can be introduced to the practice of brachytherapy for cervical cancer.

\section{Acknowledgement}

This research was presented at the World Congress of Brachytherapy held in San Francisco, CA, USA on June 27-29, 2016.

\section{Disclosure}

Authors report no conflict of interest.

\section{References}

1. Pötter R, Kirisits C, Fidarova EF et al. Present status and future of high-precision image guided adaptive brachytherapy for cervix carcinoma. Acta Oncol 2008; 47:1325-1336.

2. Viswanathan AN, Erickson BA. Seeing is saving: the benefit of 3D imaging in gynecologic brachytherapy. Gynecol Oncol 2015; 138: 207-215.

3. Pötter R, Georg P, Dimopoulos JC et al. Clinical outcome of protocol based image (MRI) guided adaptive brachytherapy combined with 3D conformal radiotherapy with or without chemotherapy in patients with locally advanced cervical cancer. Radiother Oncol 2011; 100:116-123.

4. Simpson DR, Scanderbeg DJ, Carmona R et al. Clinical outcomes of computed tomography-based volumetric brachytherapy planning for cervical cancer. Int J Radiat Oncol Biol Phys 2015; 93: 150-157.

5. Georg P, Pötter R, Georg D et al. Dose effect relationship for late side effects of the rectum and urinary bladder in magnetic resonance image-guided adaptive cervix cancer brachytherapy. Int I Radiat Oncol Biol Phys 2012; 82: 653-657.

6. Kato S, Tran DN, Ohno T et al. CT-based 3D dose-volume parameter of the rectum and late rectal complication in patients with cervical cancer treated with high-dose-rate intracavitary brachytherapy. J Radiat Res 2010; 51: 215-221.

7. Mazeron R, Fokdal LU, Kirchhener K et al. Dose-volume effect relationships for late rectal morbidity in patients treated with chemoradiation and MRI-guided adaptive brachytherapy for locally advanced cervical cancer: Results from the prospective multicenter EMBRACE study. Radiother Oncol 2016; 120: 412-419.

8. Denny L, Herrero R, Levin C et al. Cervical Cancer. In: Cancer: Disease Control Priorities. Gelband H, Jha P, Sankaranarayanan R, Horton S (eds.). $3^{\text {rd }}$ ed. Volume 3. The International Bank for Reconstruction and Development/The World Bank, Washington 2015; Chapter 4.

9. Kim H, Rajagopalan MS, Beriwal S et al. Cost-effectiveness analysis of 3D image-guided brachytherapy compared with $2 \mathrm{D}$ brachytherapy in the treatment of locally advanced cervical cancer. Brachytherapy 2015; 14: 29-36.

10. Davis BJ, Horwitz EM, Lee R et al. American Brachytherapy Society consensus guidelines for transrectal ultrasound-guided permanent prostate brachytherapy. Brachytherapy 2012; 11: 6-19.

11. Hoskin PJ, Colombo A, Henry A et al. GEC/ ESTRO recommendations on high dose rate afterloading brachytherapy for localized prostate cancer: An update. Radiother Oncol 2013; 107: 325-332.

12. Van Dyk S, Narayan K, Fisher R et al. Conformal brachytherapy planning for cervical cancer using transabdominal ultrasound. Int J Radiat Oncol Biol Phys 2009; 75: 64-70.
13. Narayan K, van Dyk S, Bernshaw D et al. Ultrasound guided conformal brachytherapy of cervix cancer: survival, patterns of failure, and late complications. J Gynecol Oncol 2014; 25: 206-213.

14. Schmid MP, Nesvacil N, Pötter R et al. Transrectal ultrasound for image-guided adaptive brachytherapy in cervix cancer - an alternative to MRI for target definition? Radiother Oncol 2016; 120: 467-472.

15. Lim K, Chan P, Dinniwell R et al. Cervical cancer regression measured using weekly magnetic resonance imaging during fractionated radiotherapy: radiobiologic modeling and correlation with tumor hypoxia. Int J Radiat Oncol Biol Phys 2008; 70: 126-133.

16. Ohara K, Tanaka YO, Oki A et al. Comparison of tumor regression rate of uterine cervical squamous cell carcinoma during external beam and intracavitary radiotherapy. Radiat Med 2008; 26: 526-532.

17. Van Dyk S, Kondalsamy-Chennakesavan S, Schneider M et al. Comparison of measurements of the uterus and cervix obtained by magnetic resonance and transabdominal ultrasound imaging to Identify the brachytherapy target in patients with cervix cancer. Int J Radiat Oncol Biol Phys 2014; 88: 860-865.

18. Petric P, Kirisits C. Potential role of TRAns Cervical Endosonography (TRACE) in brachytherapy of cervical cancer: proof of concept. J Contemp Brachytherapy 2016; 8: 215-220. 